\title{
Order-restricted Scores Test for the Evaluation of Population-based Case-control Studies when the Genetic Model is Unknown
}

\author{
Ludwig A. Hothorn \\ Leibniz Universität Hannover
}

\author{
Torsten Hothorn \\ Ludwig-Maximilians-Universität München
}

\begin{abstract}
This is an preprint of an article to be published in Biometrical Journal. Copyright (C) 2009 WILEY-VCH Verlag GmbH \& Co. KGaA, Weinheim; available online at http: //www.biometrical-journal.com.

The Cochran-Armitage linear trend test for proportions is often used for genotypebased analysis of candidate gene association. Depending on the underlying genetic mode of inheritance, the use of model-specific scores maximises the power. Commonly, the underlying genetic model, i.e., additive, dominant or recessive mode of inheritance, is a priori unknown. Association studies are commonly analysed using permutation tests, where both inference and identification of the underlying mode of inheritance are important. Especially interesting are tests for case-control studies, defined by a maximum over a series of standardised Cochran-Armitage tests, because such a procedure has power under all three genetic models.

We reformulate the test problem and propose a conditional maximum test of scoresspecific linear-by-linear association tests (Agresti 2002). For maximum-type, sum and quadratic test statistics the asymptotic expectation and covariance can be derived in a closed form and the limiting distribution is known. Both the limiting distribution and approximations of the exact conditional distribution can easily be computed using standard software packages. In addition to these technical advances, we extend the area of application to stratified designs, studies involving more than two groups and the simultaneous analysis of multiple loci by means of multiplicity-adjusted $p$-values for the underlying multiple Cochran-Armitage trend tests. The new test is applied to reanalyse a study investigating genetic components of different subtypes of psoriasis.

A new and flexible inference tool for association studies is available both theoretically as well as practically since already available software packages can be easily used to implement the suggested test procedures.
\end{abstract}

Keywords: genetic association, case-control study, robust trend test, maximum test, conditional inference.

\section{Objectives}

In population-based case-control studies the association between a candidate allele and a disease can be evaluated by the Cochran-Armitage (CA) trend test (Armitage 1955), regardless of whether or not Hardy-Weinberg equilibrium holds (Sasieni 1997). The CA test is based on a set of scores assigned to the alleles. For genotypes $a a, A a$, or $A A$, with $A$ denoting a high risk 
Table 1: Genotype distributions for cases and controls.

\begin{tabular}{llll} 
& Cases & Controls & Total \\
\hline$a a$ & $r_{a a}$ & $s_{a a}$ & $n_{a a}$ \\
$a A$ & $r_{a A}$ & $s_{a A}$ & $n_{a A}$ \\
$A A$ & $r_{A A}$ & $s_{A A}$ & $n_{A A}$ \\
Total & $R$ & $S$ & $N$ \\
\hline
\end{tabular}

candidate allele and $a$ any of the other alleles, three-dimensional score vectors optimising the power of the CA test against dominant, additive, and recessive alternatives can be defined. If the underlying mode of inheritance is known, the choice of an appropriate score vector for the trend test is obvious. However, in situations where the underlying genetic model is unknown choosing the wrong score vector leads to a substantial loss of power as shown by Freidlin, Zheng, Li, and Gastwirth (2002). Therefore, inference procedures with good power under all three genetic models are of special interest. An appealing approach is to construct a test based on all three possible trend tests, for example utilising the maximum of the standardised test statistics of the CA tests which are optimal under the dominant, additive, and recessive model. An unconditional version of this test was proposed and investigated by Freidlin et al. (2002). The distribution of this maximum test, called MAX test hereafter, under the null hypothesis of equal genotype distribution in cases and controls is approximated by simulation procedures by Freidlin et al. (2002) since the unconditional asymptotic distribution is hard to derive.

In this paper, we show that the conditional MAX test is a simple special case of a general class of linear statistics whose conditional distribution is easy to compute. Thus, without additional theoretical effort, the MAX test enjoys all the nice properties of this class of conditional tests. Therefore, we embed the MAX test as suggested by Freidlin et al. (2002) into the flexible framework for conditional independence tests introduced by Strasser and Weber (1999). The merits of doing so are i) the conditional reference distribution for the MAX test can be easily approximated either by evaluating a three-dimensional normal distribution or by conditional Monte Carlo experiments, ii) tests for stratified designs, designs with more than two groups and two or more loci can be defined in a rather straightforward way, iii) the most likely underlying mode of inheritance can be estimated by multiplicity-adjusted $p$-values for the three CA statistics under consideration, iv) the simultaneous analysis of multiple loci, and v) the analysis of genetic association studies using the MAX test and its newly introduced extension can be performed by already available software implementations of the Strasser and Weber (1999) framework.

\section{Maximum Test}

For case-control studies investigating the association between a binary phenotype and the measured alleles of a candidate gene the data are typically presented as empirical genotype distribution in each group. For a simple bi-allelic marker the data can be presented in a $3 \times 2$ contingency table, where $A$ is the high risk candidate allele and $a$ is any of the other alleles (see Table 1). 
Table 2: Genotype distribution reformulated.

\begin{tabular}{lllc|cccc}
$i$ & $\mathbf{Y}_{i}$ & $\mathbf{X}_{i}$ & $w_{i}$ & $h\left(\mathbf{Y}_{i}\right)$ & $g_{\text {add }}\left(\mathbf{X}_{i}\right)$ & $g_{\text {dom }}\left(\mathbf{X}_{i}\right)$ & $g_{\text {rec }}\left(\mathbf{X}_{i}\right)$ \\
\hline 1 & Case & $a a$ & $r_{a a}$ & 1 & 0 & 0 & 0 \\
2 & Case & $A a$ & $r_{a A}$ & 1 & 1 & 1 & 0 \\
3 & Case & $A A$ & $r_{A A}$ & 1 & 2 & 1 & 1 \\
4 & Control & $a a$ & $s_{a a}$ & 0 & 0 & 0 & 0 \\
5 & Control & $A a$ & $s_{a A}$ & 0 & 1 & 1 & 0 \\
6 & Control & $A A$ & $s_{A A}$ & 0 & 2 & 1 & 1 \\
\hline
\end{tabular}

We are interested in a comparison of the genotype distributions, i.e., the penetrances $f_{j}=$ $P(\operatorname{case} \mid j)$ for $j \in\{a a, a A, A A\}$ :

$$
H_{0}: f_{a a}=f_{a A}=f_{A A} \text { vs. } H_{1}: f_{a a} \leq f_{a A} \leq f_{A A}
$$

where at least one of the inequalities in the alternative is strict.

The CA test statistic with scores $\xi=\left(\xi_{a a}, \xi_{a A}, \xi_{A A}\right)$ is essentially (modulo standardisation)

$$
\mathrm{CA}(\xi)=\sum_{j \in\{a a, a A, A A\}} \xi_{j} r_{j} .
$$

If the mode of inheritance is best described by the dominant model, the scores $\xi_{\text {dom }}=(0,1,1)$ $\left(f_{a a}<f_{a A}=f_{A A}\right)$ will lead to a trend test with maximal power. Under the recessive model the score vector $\xi_{\text {rec }}=(0,0,1)\left(f_{a a}=f_{a A}<f_{A A}\right)$ is power optimal whereas a linear trend represented by scores $\xi_{\text {add }}=(0,1,2)$ should be chosen when the mode if inheritance is additive, i.e., $f_{a a}<f_{a A}<f_{A A}$ (Sasieni 1997; Slager and Schaid 2001). However, the underlying genetic model is rarely known a priori. Motivated by the problem of choosing the 'right' score vector, Freidlin et al. (2002) proposed the MAX unconditional test as the maximum of three standardised CA tests with scores $\xi_{\text {dom }}, \xi_{\text {add }}$, and $\xi_{\text {rec }}$ as a global test for association. A similar approach (Zheng, Freidlin, Li, and Gastwirth 2003; Zheng 2008) is to introduce a parameter $\eta$ for the score vector $\xi_{\eta}=(0, \eta, 1)$ and to choose $\eta$ in a data-driven way. This procedure (based on a grid of $\eta$ values) is a special case of the general framework described in the following, see Appendix A for more details.

In the following, we reformulate the test problem and embed the MAX test into a general framework for conditional inference procedures, derive its limiting distribution and propose extensions to stratified designs, more than two groups and multiple loci.

\subsection{Reformulation of the Problem}

Let $\mathbf{Y}_{i}$ denote the case and control status and $\mathbf{X}_{i}$ the genotype for all cells $i=1, \ldots, n=$ 6 . The weights $w_{i}$ represent the number of observations in each cell with total number of observations $N=\sum_{i} w_{i}$. The influence function $h$ provides us with a zero-one dummy coding of the groups (being one for cases and zero for controls). Moreover, three transformations $g$ of the genotype are under test: $g_{\text {dom }}$ assigns scores $\xi_{\text {dom }}$ to genotypes ( $\left.a a, A a, A A\right), g_{\text {add }}$ assigns scores $\xi_{\text {add }}$ and $g_{\text {rec }}$ implements scores $\xi_{\text {rec }}$, cf. Table 2. 


\subsection{Inference Problem and Linear Statistic}

We are interested in testing the null hypothesis of independence of grouping $\mathbf{Y}$ and genotype $\mathbf{X}$, i.e., the equality of the distribution $D(\mathbf{Y})$ to the conditional distribution $D(\mathbf{Y} \mid \mathbf{X})$ of $\mathbf{Y}$ given $\mathbf{X}$

$$
H_{0}: D(\mathbf{Y} \mid \mathbf{X})=D(\mathbf{Y})
$$

against ordered alternatives. First, we define a three-dimensional statistic $\mathbf{T}$, each dimension being associated with one of the scores $g_{\text {add }}, g_{\text {dom }}$, and $g_{\text {rec }}$. Each statistic is defined by the sum of the scores multiplied by the weights associated with cases, i.e., is equivalent to the Cochran-Armitage statistic (1):

$$
\mathbf{T}=\left(\mathbf{T}_{\text {add }}, \mathbf{T}_{\text {dom }}, \mathbf{T}_{\text {rec }}\right)=\sum_{i=1}^{n} w_{i} g\left(\mathbf{X}_{i}\right) h\left(\mathbf{Y}_{i}\right) \in \mathbb{R}^{3}
$$

with $g\left(\mathbf{X}_{i}\right)=\left(g_{\text {add }}\left(\mathbf{X}_{i}\right), g_{\text {dom }}\left(\mathbf{X}_{i}\right), g_{\text {rec }}\left(\mathbf{X}_{i}\right)\right)$. Thus, the three-dimensional linear statistic $\mathbf{T}$ is the vector of the unstandardised Cochran-Armitage statistics $\left(\mathrm{CA}\left(\xi_{\text {dom }}\right), \mathrm{CA}\left(\xi_{\text {add }}\right), \mathrm{CA}\left(\xi_{\text {rec }}\right)\right)$ for the dominant, additive, and recessive model.

\subsection{Conditional Expectation and Covariance}

The distribution of $\mathbf{T}$ depends on the joint distribution of $\mathbf{Y}$ and $\mathbf{X}$, which is unknown under almost all practical circumstances. At least under the null hypothesis one can dispose of this dependency by fixing the genotypes and conditioning on all possible permutations of the groups. This principle leads to test procedures known as permutation tests. Strasser and Weber (1999) derived closed-form expressions for the conditional expectation $\mu \in \mathbb{R}^{p q}$ and covariance $\Sigma \in \mathbb{R}^{3 \times 3}$ of $\mathbf{T}$ under $H_{0}$ given all permutations of the groupings.

The conditional expectation of the influence function $h$ is

$$
\mathrm{E}(h)=N^{-1} \sum_{i} w_{i} h\left(\mathbf{Y}_{i}\right) \in \mathbb{R}
$$

with corresponding variance

$$
\mathbb{V}(h)=N^{-1} \sum_{i} w_{i}\left(h\left(\mathbf{Y}_{i}\right)-\mathrm{E}(h)\right)^{2} .
$$

The conditional expectation of the linear statistic $\mathbf{T}$ is

$$
\begin{aligned}
\mu & =\mathrm{E}(\mathbf{T})=\mathrm{E}(h) \sum_{i=1}^{n} w_{i} g\left(\mathbf{X}_{i}\right), \\
\Sigma & =\mathbb{V}(\mathbf{T}) \\
& =\frac{N}{N-1} \mathbb{V}(h) \times\left(\sum_{i} w_{i}\left(g\left(\mathbf{X}_{i}\right) g\left(\mathbf{X}_{i}\right)^{\top}\right)\right) \\
& -\frac{1}{N-1} \mathbb{V}(h) \times\left(\sum_{i} w_{i} g\left(\mathbf{X}_{i}\right)\right)\left(\sum_{i} w_{i} g\left(\mathbf{X}_{i}\right)\right)^{\top} .
\end{aligned}
$$

The three-dimensional expectation $\mu$ and the three diagonal elements of the covariance matrix $\Sigma$ contain the mean and the variances for the additive, dominant and recessive (unstandardised) Cochran-Armitage statistics under $H_{0}$, as given in (1) and (2), respectively. 
Note that the complete covariance structure, and thus the correlation between the elements of the three-dimensional statistic $\mathbf{T}$, is known and can be computed for the data at hand. The corresponding correlation matrix coincides with the correlations obtained for the three CA test statistics by Freidlin et al. (2002).

\subsection{Test Statistics}

Based on the three-dimensional statistic $\mathbf{T}$ and its expectation $\mu$ and covariance matrix $\Sigma$, we can easily construct test statistics and derive their distribution under the conditions described in the null hypothesis. As the number of observations $N$ tends to infinity, Strasser and Weber (1999) proved that the limiting distribution of the three-dimensional statistic $\mathbf{T}$ is a threedimensional normal distribution with expectation $\mu$ and covariance $\Sigma$. Thus, the asymptotic distribution of a maximum-type statistic

$$
c_{\max }(\mathbf{T}, \mu, \Sigma)=\max \left|\frac{\mathbf{T}-\mu}{\operatorname{diag}(\Sigma)^{1 / 2}}\right|
$$

can be evaluated by computing three-dimensional normal probabilities. Here, $\operatorname{diag}(\Sigma)^{1 / 2}$ are the conditional standard deviations of the elements of $\mathbf{T}$. Alternatively, either the sum (or average) statistic

$$
c_{\text {sum }}=\frac{\mathbf{1}^{\top} \mathbf{T}-\mathbf{1}^{\top} \mu}{\mathbf{1}^{\top} \Sigma \mathbf{1}}, \quad \mathbf{1}=(1,1,1)^{\top}
$$

or a quadratic form based on the Moore-Penrose inverse $\Sigma^{+}$of the conditional covariance matrix $\Sigma$, i.e.,

$$
c_{\text {quad }}(\mathbf{T}, \mu, \Sigma)=(\mathbf{T}-\mu)^{\top} \Sigma^{+}(\mathbf{T}-\mu)
$$

can be used and follow a standard normal or a $\chi^{2}$ distribution with two degrees of freedom, respectively. The sum and quadratic form statistics, which are competitors for the MAX test statistic, reveal high power for an average alternative while the maximum-type form for a particular genetic alternative. In addition, simple linear combinations of the standardised optimum tests for each of the models have been proposed (Gastwirth 1985). Often the most appropriate test is not the average of all these tests but a linear combination of a few "extreme" members. Nonlinear MAX type tests have greater efficiency robustness properties when the minimum correlation between the optimal tests for the models considered is less than .70 (Freidlin, Podgor, and Gastwirth 1999), therefore, we focus on the MAX test. A further advantage of maximum-type statistics is that information about the likely genetic model underlying the data is available using multiplicity-adjusted $p$-values. Note that, under any circumstances, the exact conditional distribution can be approximated by conditional Monte Carlo methods, which is especially attractive for small sample sizes $N$ when we can not expect asymptotics to work well.

\subsection{Illustration}

In order to compare the conditional test and its implementation with the unconditional results reported by Freidlin et al. (2002), we reanalyse a study on the association between a variant of the epidermal growth factor (EGF) gene and malignant melanoma according to 
Table 3: Melanoma data.

\begin{tabular}{llll} 
& In situ & Control & Total \\
\hline AA & 6 & 32 & 38 \\
AG & 8 & 47 & 55 \\
GG & 10 & 20 & 30 \\
Total & 24 & 99 & 123 \\
\hline
\end{tabular}

Table 4: MAX test for Melanoma data with linear statistic T, its conditional expectation $\mu$, standard deviation $\sigma$ and corresponding standardised statistic along with multiplicityadjusted $p$-values.

\begin{tabular}{lllllll} 
& $\mathbf{T}$ & $\mu$ & $\sigma$ & $(\mathbf{T}-\mu) / \sigma$ & $p_{\text {asympt }}$ & $p_{\text {step-down }}$ \\
\hline dominant & 18 & 16.5854 & 2.0391 & 0.6938 & 0.3907 & 0.3302 \\
additive & 28 & 22.4390 & 3.2687 & 1.7013 & 0.0868 & 0.0654 \\
recessive & 10 & 5.8537 & 1.8951 & 2.1879 & 0.0303 & 0.0359 \\
\hline
\end{tabular}

Shahbazi, Pravica, Nasreen, Fakhoury, Fryer, Strange, Hutchinson, Osborne, Lear, Smith, and Hutchinson (Table 3, 2002).

The linear statistic $\mathbf{T}$, its conditional expectation $\mu$, the standard deviations $\sigma=\sqrt{\operatorname{diag}(\Sigma)}$, and the corresponding standardised CA statistics are given in Table 4. In addition, we immediately are provided with the covariance matrix

$$
\Sigma=\left(\begin{array}{rrr}
4.1579 & 5.6255 & 1.4675 \\
5.6255 & 10.6845 & 5.0590 \\
1.4675 & 5.0590 & 3.5915
\end{array}\right)
$$

and corresponding correlation matrix

$$
\operatorname{cor}(\Sigma)=\left(\begin{array}{lll}
1.0000 & 0.8440 & 0.3798 \\
0.8440 & 1.0000 & 0.8167 \\
0.3798 & 0.8167 & 1.0000
\end{array}\right)
$$

These values are similar to the correlations between the three different CA test statistics as reported by Freidlin et al. (2002).

The MAX test has a test statistic equal to 2.1879 and its asymptotic $p$-value is 0.0303 (the minimum of $p_{\text {asympt }}$ in Table 4 ) which is roughly the same $p$-value as shown in Table 8 of Freidlin et al. (2002). However, this global $p$-value does not give any information about the underlying genetic model. Multiplicity-adjusted $p$-values $\left(p_{\text {asympt }}\right.$ in Table 4 ) for each of the dominant, additive, and recessive tests indicate which mode of inheritance describes the data best (see Section 4 in addition): These results suggest that the cancer follows a recessive model, although an additive or intermediate model cannot be ruled out based on these data. We might want to check whether the asymptotic approximation work well enough in this situation. The exact conditional $p$-value is approximated by a conditional Monte Carlo procedure 
with 49999 random permutations of the data and the corresponding step-down multiplicityadjusted $p$-values (Westfall and Young 1993) are given as $p_{\text {step-down }}$ in Table 4 . The small differences between the asymptotic and approximated $p$-values indicates that using the asymptotic distribution is adequate.

\subsection{Generalisations}

A straightforward generalisation is the consideration of $3 \times k$ tables instead of $3 \times 2$ tables, where sub-types of cases are compared with a control. For example, the genotype distribution of the control can compare the genotype of cases with early and late onset of a certain disease. A score can be attached to each group, for example 1 to the control group and $-1 / 2$ for both the early and late onset cases leading to a linear-by-linear association test. Alternatively, a trend in the onset of the disease can be described by scores $0,1,2$ for the three groups.

In stratified designs, only permutations within each stratum, gender or family history, are admissible; therefore, the expectation $\mu$ and covariance $\Sigma$ has to be computed separately for each stratum and is then aggregated over all possible strata. A special version of a stratified design is the commonly used meta-analysis, including several independent studies as strata, see e.g. Kavvoura and Ioannidis (2008). In the absence of a a priori assumption for a particular mode of inheritance, recently Salanti and Higgins (2008) proposed a Bayesian approach.

In a recent meta-analysis of genome-wide association studies variants on chromosome 9p21.3 were identified affecting the coronary artery disease (Schunkert, Gotz, Braund, McGinnis, Tregouet, Mangino, Linsel-Nitschke, Cambien, Hengstenberg, Stark, Blankenberg, Tiret, Ducimetiere, Keniry, Ghori, Schreiber, El Mokhtari, Hall, Dixon, Goodall, Liptau, Pollard, Schwarz, Hothorn, Wichmann, Konig, Fischer, Meisinger, Ouwehand, Deloukas, Thompson, Erdmann, Ziegler, and Samani 2008) where all seven studies revealed the same additive mode of inheritance using the here proposed approach.

Finally, it is interesting to consider multiple loci, i.e., multiple genotype distributions, simultaneously. For two loci, we can look at all six CA tests by defining a linear statistic $\mathbf{T}$ containing the three CA tests for the first as well as the three CA tests for the second locus. As a consequence, we can compute the complete covariance matrix and take the underlying correlations between the two loci as well as between the three genetic models into account.

\section{Illustration and Application}

Reich, Mössner, König, Westphal, Ziegler, and Neumann (2002) investigate the association between psoriasis and polymorphisms of genes encoding tumour necrosis Factor- $\alpha$ and Interleukin- $1 \beta$ where for the IL1B_511 locus the related $3 \times 2$ table data are given in Table 5 . A control group and two groups of affected people with early and late onset of the disease are under test. One is interested in detecting any deviation from independence of genotype distribution for both loci and the three groups in either females and / or males. Attaching scores $1,-1 / 2,-1 / 2$ to the control, early and late onset group results in a linear statistic $\mathbf{T}$ with six elements: three models for each of the two loci.

The multiplicity-adjusted $p$-values in Table 6 indicate that there is a strong deviation from independence for the TNFA_238 locus. The recessive model has the largest $p$-value and thus it is not likely that this model is true. The $p$-values for the dominant and the additive are extremely small, so either of these models could have generated the data. We can simulta- 
Table 5: Psoriasis data

IL1B_511 locus

\begin{tabular}{llllll} 
Male & & Control & Early Onset & Late Onset & Total \\
\hline & CC & 75 & 54 & 29 & 158 \\
& CT & 93 & 44 & 13 & 150 \\
& TT & 14 & 7 & 4 & 25 \\
& Total & 182 & 105 & 46 & 333 \\
Female & & Control & Early Onset & Late Onset & Total \\
\hline & CC & 76 & 26 & 17 & 119 \\
& CT & 69 & 20 & 10 & 99 \\
& TT & 18 & 5 & 2 & 25 \\
& Total & 163 & 51 & 29 & 243
\end{tabular}

TNFA_238 locus

\begin{tabular}{llllll} 
Male & & Control & Early Onset & Late Onset & Total \\
\hline & GG & 170 & 71 & 40 & 281 \\
& GA & 12 & 33 & 6 & 51 \\
& AA & 0 & 1 & 0 & 1 \\
& Total & 182 & 105 & 46 & 333 \\
Female & & Control & Early Onset & Late Onset & Total \\
\hline & GG & 146 & 43 & 24 & 213 \\
& GA & 17 & 8 & 5 & 30 \\
AA & 0 & 0 & 0 & 0 \\
& Total & 163 & 51 & 29 & 243 \\
\hline
\end{tabular}

neously reject the null hypothesis of independence between the genotype distribution of the IL1B_511 locus and the three groups. Here, the dominant model seems to explain the data best.

Our analysis improves upon the original analysis of these data by Reich et al. (2002) with respect to three points: All three groups and the stratification by gender are taken into account and and the new test makes use of the correlation between the two loci instead of applying a Bonferroni correction in order to maintain an overall significance level.

Recently, Bagos and Nikolopoulos (2007) proposed a penalty-free selection approach for the underlying mode of inheritance based on the parameter estimates in a logistic regression model. Here, we re-analyse their meta-analysis data on the association of KIR6.2 gene polymorphism with type II diabetes (Table 1 on page 3 in Bagos and Nikolopoulos 2007).

\section{Simulation Experiments}

It might be questioned if the minimal $p$-value can be observed for the correct mode of in- 
Table 6: MAX test for psoriasis data: Asymptotic multiplicity-adjusted $p$-values.

\begin{tabular}{lrr} 
& TNFA_238 & IL1B_511 \\
\hline dominant & $<0.0001$ & 0.0407 \\
additive & $<0.0001$ & 0.1051 \\
recessive & 0.7241 & 0.9819 \\
\hline
\end{tabular}

Table 7: MAX test for Type II diabetes data with linear statistic $\mathbf{T}$, its conditional expectation $\mu$, standard deviation $\sigma$ and corresponding standardised statistic along with multiplicityadjusted $p$-values.

\begin{tabular}{lllllll} 
& $\mathbf{T}$ & $\mu$ & $\sigma$ & $(\mathbf{T}-\mu) / \sigma$ & $p_{\text {asympt }}$ & $p_{\text {step-down }}$ \\
\hline dominant & 337 & 320.4287 & 7.0531 & 2.3495 & 0.0207 & 0.0112 \\
additive & 438 & 404.0265 & 10.1618 & 3.3433 & 0.0012 & 0.001 \\
recessive & 101 & 83.5979 & 5.2912 & 3.2889 & 0.0012 & 0.0013 \\
\hline
\end{tabular}

heritance with high probability and thus how good the 'estimator' is under practical circumstances. The frequency of correct model identifications and the power of the MAX test is investigated in some simple situations in the following.

Many different patterns of penetrances $f_{j}, j \in\{a a, a A, A A\}$, disease prevalence $p$, sample size of cases and controls $R, S$ can be investigated in a simulation study. We will focus on a high prevalent disease (i.e., $p=0.5$ ), penetrances according to an additive, recessive and dominant genetic model (as well as no association characterising the null hypothesis) for a total sample size of $N=400$ divided into the balanced $R=S=200$ and several unbalanced sampling schemes. Unbalanced data are of interest because real data examples exist with seriously higher control sample size, see e.g. the data in Table 3, or with more cases, see e.g. the IL13 polymorphism in atopic dermatitis Neuhäuser (2002), Table 4. For the proposed MAX test both the global power $\pi_{\text {global }}$ (the decision rate in favour of any alternative) and the correct model identification rates $\psi_{\text {add }}, \psi_{\text {rec }}, \psi_{\text {dom }}$ are compared with the power of the individual genetic model tests $\pi_{\mathrm{add}}, \pi_{\mathrm{rec}}, \pi_{\mathrm{dom}}$ in Table 8 .

Per definition all tests control the type I error rates. Clearly, the power is maximal for the individual, unadjusted tests when the genetic model is known (bold marked). But the a priori knowledge of the genetic model is commonly unrealistic. For balanced samples sizes the power of the MAX test is independent of the underlying genetic model and marginal smaller compared with the maximum power for the known model. Additionally to the global decision that a significant association exists, the MAX test provide an adjusted $p$-value for the most likely genetic model. In this case, the identification of the additive model is most difficult because of the two equal competitors; whereas the identification of the dominant or recessive model is easier because the additive model is the only competitor. For unbalanced designs the power decreases although the total sample size remains constant. 
Table 8: Type I error rate and empirical power estimates $(\pi)$ for prevalence $p=0.5$ based on 10000 runs with sample sizes $R$ (cases) and $S$ (controls) along with correct model identification rates $\psi$.

\begin{tabular}{llll|lll|lll} 
Model & $\mathrm{R}$ & $\mathrm{S}$ & $\pi_{\text {global }}$ & $\psi_{\text {add }}$ & $\psi_{\text {rec }}$ & $\psi_{\text {dom }}$ & $\pi_{\text {add }}$ & $\pi_{\text {rec }}$ & $\pi_{\text {dom }}$ \\
\hline Null & 200 & 200 & 0.048 & 0.012 & 0.017 & 0.019 & 0.051 & 0.047 & 0.049 \\
dominant & 200 & 200 & 0.85 & 0.13 & 0.01 & 0.71 & 0.75 & 0.23 & $\mathbf{0 . 9 1}$ \\
additive & 200 & 200 & 0.84 & 0.53 & 0.10 & 0.21 & $\mathbf{0 . 8 8}$ & 0.71 & 0.78 \\
recessive & 200 & 200 & 0.86 & 0.16 & 0.69 & 0.01 & 0.80 & $\mathbf{0 . 9 1}$ & 0.28 \\
dominant & 100 & 300 & 0.72 & 0.16 & 0.01 & 0.55 & 0.63 & 0.21 & $\mathbf{0 . 8 0}$ \\
additive & 100 & 300 & 0.73 & 0.43 & 0.14 & 0.16 & $\mathbf{0 . 7 8}$ & 0.60 & 0.65 \\
recessive & 100 & 300 & 0.77 & 0.16 & 0.60 & 0.01 & 0.67 & $\mathbf{0 . 8 2}$ & 0.22 \\
dominant & 300 & 100 & 0.76 & 0.11 & 0.01 & 0.64 & 0.66 & 0.19 & $\mathbf{0 . 8 2}$ \\
additive & 300 & 100 & 0.75 & 0.42 & 0.09 & 0.24 & $\mathbf{0 . 7 9}$ & 0.59 & 0.69 \\
recessive & 300 & 100 & 0.75 & 0.17 & 0.55 & 0.01 & 0.69 & $\mathbf{0 . 8 2}$ & 0.24 \\
\hline
\end{tabular}

\section{Computational Details}

The coin add-on package (Hothorn, Hornik, van de Wiel, and Zeileis 2006, 2008, 2009) to the R system for statistical computing ( $\mathrm{R}$ Development Core Team 2009) provides an implementation of the conditional inference framework sketched in this section. The analysis of an association study by the MAX test only requires the user to set-up the score function $g$. Then, the function independence_test can be used to perform the MAX test and to compute multiplicity-adjusted $p$-values.

\section{Conclusions}

We propose a flexible approach to permutation tests for association of a bi-allelic marker with a disease in population-based case-control studies. The joint conditional asymptotic distribution of the three underlying linear association tests, i.e., Cochran-Armitage tests with optimal scores for additive, dominant, and recessive modes of inheritance, is known and can be used to approximate the distribution of the corresponding maximum statistic. Not only a global $p$-value can be derived this way but also multiplicity-adjusted $p$-values for each of the individual models. When the mode of inheritance is unknown, remarkably high correct model selection rates can be achieved. Based on a general framework for conditional inference we extend the MAX test to stratified designs, $3 \times k$ tables as well as multiple loci. Correlations between loci and corresponding association tests are taken into account leading to more powerful multiple test procedure. Further modifications and extensions and specific choices of $g$ and $h$ are described by Hothorn et al. (2006) and Hothorn et al. (2008). For small sample sizes, a better approximation of the $p$-values can be obtained from Monte Carlo resampling techniques. For genome-wide association studies, computing time is a limiting factor; a further advantage for the above approach (Ziegler, Koenig, and Thompson 2008).

The proposed procedures are easily applicable using the computational tools provided by the $\mathrm{R}$ add-on package coin as illustrated in Appendix A. A future modification will be the use of 
model-specific genomic-control corrected tests analogously to Zang, Zhang, Yang, and Zheng (2007) in the possible case of population stratification.

\section{Acknowledgements}

We would like to thank Andreas Ziegler and Inke R. Koenig for providing us with the psoriasis data.

\section{References}

Agresti A (2002). Categorical Data Analysis. 2nd edition. John Wiley \& Sons, Hoboken, New Jersey.

Armitage P (1955). "Tests for Linear Trends in Proportions and Frequencies." Biometrics, 11(3), 375-386.

Bagos P, Nikolopoulos G (2007). "A method for meta-analysis of case-control genetic association studies using logistic regression." Statistical Applications in Genetics and Molecular Biology, 6, 17.

Freidlin B, Podgor M, Gastwirth J (1999). "Efficiency Robust Tests for Survival or Ordered Categorical Data." Biometrics, 55(3), 883-886.

Freidlin B, Zheng G, Li Z, Gastwirth J (2002). "Trend tests for case-control studies of genetic markers: Power, sample size and robustness." Human Heredity, 53(3), 146-152.

Gastwirth JL (1985). "The Use of Maximin Efficiency Robust Tests in Combining Contingency Tables and Survival Analysis." Journal of the American Statistical Association, 80(390), $380-384$.

Hothorn T, Hornik K, van de Wiel MA, Zeileis A (2006). "A Lego System for Conditional Inference." The American Statistician, 60(3), 257-263. doi:10.1198/000313006X118430.

Hothorn T, Hornik K, van de Wiel MA, Zeileis A (2008). "Implementing a Class of Permutation Tests: The coin Package." Journal of Statistical Software, 28(8), 1-23. doi: 10.18637/jss.v028.i08.

Hothorn T, Hornik K, van de Wiel MA, Zeileis A (2009). coin: Conditional Inference Procedures in a Permutation Test Framework. R package version 1.0-2, URL http: //CRAN.R-project. org/package=coin.

Kavvoura FK, Ioannidis JPA (2008). "Methods for meta-analysis in genetic association studies: a review of their potential and pitfalls." Human Genetics, 123(1), 1-14.

Neuhäuser M (2002). "Exact tests for the analysis of case-control studies of genetic markers." Human Heredity, 54(3), 151-156.

R Development Core Team (2009). R: A Language and Environment for Statistical Computing. R Foundation for Statistical Computing, Vienna, Austria. ISBN 3-900051-00-3, URL http://www.R-project.org.

Reich K, Mössner R, König IR, Westphal G, Ziegler A, Neumann C (2002). "Promoter Polymorphisms of the Genes Encoding Tumor Necrosis Factor- $\alpha$ and Interleukin- $1 \beta$ are Associated with Different Subtypes of Psoriasis Characterized by Early and Late Disease Onset." The Journal of Investigative Dermatology, 118(1), 155-163. 
Salanti G, Higgins JPT (2008). "Meta-analysis of genetic association studies under different inheritance models using data reported as merged genotypes." Statistics in Medicine, 27(5), 764-777.

Sasieni P (1997). "From genotypes to genes: Doubling the sample size." Biometrics, 53(4), $1253-1261$.

Schunkert H, Gotz A, Braund P, McGinnis R, Tregouet DA, Mangino M, Linsel-Nitschke P, Cambien F, Hengstenberg C, Stark K, Blankenberg S, Tiret L, Ducimetiere P, Keniry A, Ghori MJR, Schreiber S, El Mokhtari NE, Hall AS, Dixon RJ, Goodall AH, Liptau H, Pollard H, Schwarz DF, Hothorn LA, Wichmann HE, Konig IR, Fischer M, Meisinger C, Ouwehand W, Deloukas P, Thompson JR, Erdmann J, Ziegler A, Samani NJ (2008). "Repeated replication and a prospective meta-analysis of the association between chromosome 9p21.3 and coronary artery disease." Circulation, 117(13), 1675-1684.

Shahbazi M, Pravica V, Nasreen N, Fakhoury H, Fryer A, Strange R, Hutchinson P, Osborne J, Lear J, Smith A, Hutchinson I (2002). "Association between functional polymorphism in EGF gene and malignant melanoma." The Lancet, 359(9304), 397-401.

Slager S, Schaid D (2001). "Case-control studies of genetic markers: Power and sample size approximations for Armitage's test for trend." Human Heredity, 52(3), 149-153.

Strasser H, Weber C (1999). "On the asymptotic theory of permutation statistics." Mathematical Methods of Statistics, 8, 220-250.

Westfall P, Young S (1993). Resampling-based Multiple Testing. John Wiley \& Sons, New York.

Zang Y, Zhang H, Yang Y, Zheng G (2007). "Robust genomic control and robust delta centralization tests for case-control association studies." Human Heredity, 63(3-4), 187195.

Zheng G (2008). "Analysis of Ordered Categorical Data: Two Score-Independent Approaches." Biometrics, 64(4), 1276-1279.

Zheng G, Freidlin B, Li Z, Gastwirth J (2003). "Choice of scores in trend tests for case-control studies of candidate-gene associations." Biometrical Journal, 45(3), 335-348.

Ziegler A, Koenig I, Thompson J (2008). "Biostatistical aspects of genome-wide association studies." Biometrical Journal, 50, 8-28. 


\section{A. Example Analyses}

The Melanoma data are represented by a table object in $\mathrm{R}$ as follows:

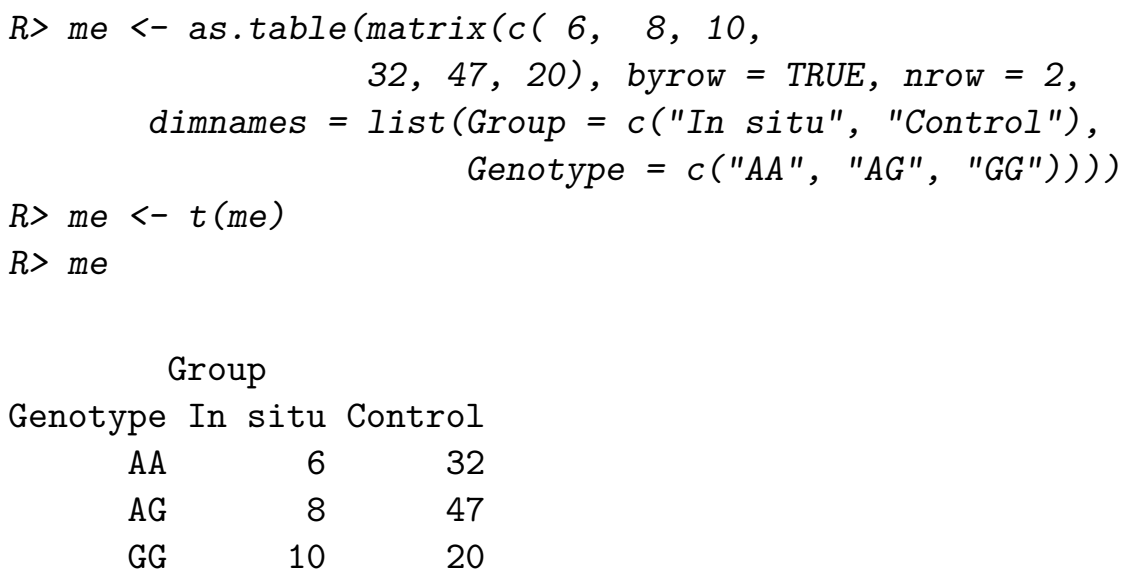

The function $g$ is implemented by the following function:

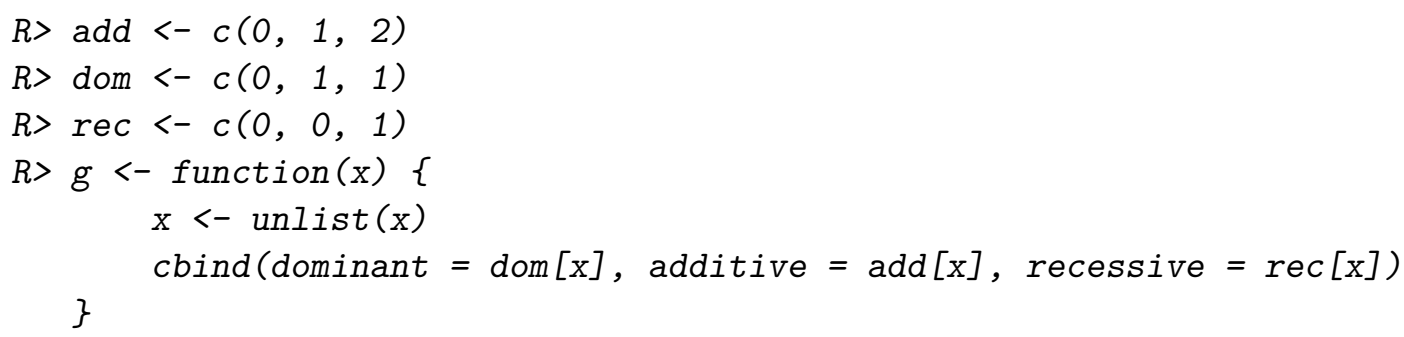

which then sets up the MAX test for the Melanoma data:

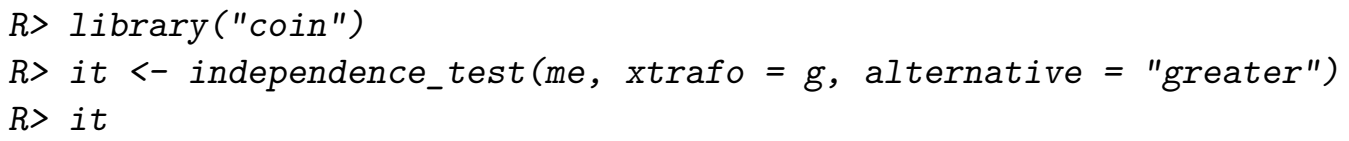

The multiplicity-adjusted $p$-values for both inference and estimating the underlying mode of inheritance are computed via:

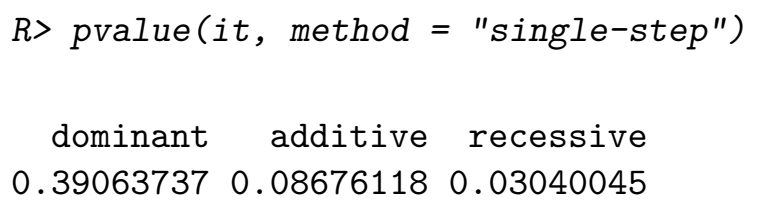


The score independent approach by Zheng (2008) maximises the Cochran-Armitage statistic over potential score vectors $\xi_{\eta}=(0, \eta, 1)$, i.e., one is interested in the test statistic $c_{\max }$ where the maximium is taken over a grid of $\eta$ values. This procedure can be performed by choosing appropriate transformation $g$, i.e., a grid of $\eta$ values in $[0,1]$

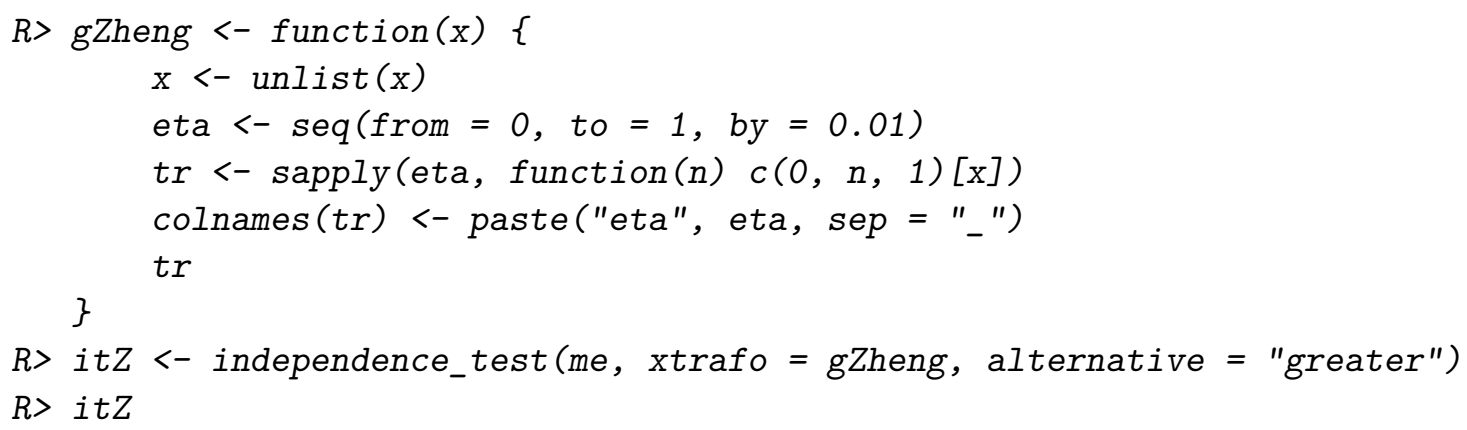

Asymptotic General Independence Test

data: Group by Genotype (AA, AG, GG)

$\operatorname{maxT}=2.1879, \mathrm{p}$-value $=0.03151$

alternative hypothesis: greater

$R>$ pvalue (itZ, method = "single-step")

$\begin{array}{rrrrr}\text { eta_0 } & \text { eta_0.01 } & \text { eta_0.02 } & \text { eta_0.03 } & \text { eta_0.04 } \\ 0.03151304 & 0.03162379 & 0.03176212 & 0.03191972 & 0.03209197 \\ \text { eta_0.05 } & \text { eta_0.06 } & \text { eta_0.07 } & \text { eta_0.08 } & \text { eta_0.09 } \\ 0.03228663 & 0.03250561 & 0.03274823 & 0.03301281 & 0.03330016 \\ \text { eta_0.1 } & \text { eta_0.11 } & \text { eta_0.12 } & \text { eta_0.13 } & \text { eta_0.14 } \\ 0.03361386 & 0.03395610 & 0.03432383 & 0.03472546 & 0.03515723 \\ \text { eta_0.15 } & \text { eta_0.16 } & \text { eta_0.17 } & \text { eta_0.18 } & \text { eta_0.19 } \\ 0.03561464 & \text { 0.03610973 } & \text { 0.03663956 } & \text { 0.03720648 } & 0.03781138 \\ \text { eta_0.2 } & \text { eta_0.21 } & \text { eta_0.22 } & \text { eta_0.23 } & \text { eta_0.24 } \\ 0.03845817 & 0.03914792 & 0.03987640 & \text { 0.04065208 } & 0.04147678 \\ \text { eta_0.25 } & \text { eta_0.26 } & \text { eta_0.27 } & \text { eta_0.28 } & \text { eta_0.29 } \\ 0.04235435 & 0.04328269 & 0.04426266 & 0.04530451 & 0.04640300 \\ \text { eta_0.3 } & \text { eta_0.31 } & \text { eta_0.32 } & \text { eta_0.33 } & \text { eta_0.34 } \\ 0.04756671 & 0.04879473 & 0.05009090 & 0.05145841 & 0.05289975 \\ \text { eta_0.35 } & \text { eta_0.36 } & \text { eta_0.37 } & \text { eta_0.38 } & \text { eta_0.39 } \\ 0.05441897 & 0.05601775 & 0.05770110 & 0.05946786 & 0.06132908 \\ \text { eta_0.4 } & \text { eta_0.41 } & \text { eta_0.42 } & \text { eta_0.43 } & \text { eta_0.44 } \\ 0.06327947 & 0.06533041 & 0.06747833 & 0.06973053 & 0.07209088 \\ \text { eta_0.45 } & \text { eta_0.46 } & \text { eta_0.47 } & \text { eta_0.48 } & \text { eta_0.49 } \\ 0.07456038 & 0.07714360 & 0.07984304 & 0.08266065 & 0.08560310 \\ \text { eta_0.5 } & \text { eta_0.51 } & \text { eta_0.52 } & \text { eta_0.53 } & \text { eta_0.54 } \\ 0.08867050 & 0.09186639 & 0.09519253 & 0.09865287 & 0.10224856 \\ \text { eta_0.55 } & \text { eta_0.56 } & \text { eta_0.57 } & \text { eta_0.58 } & \text { eta_0.59 } \\ 0.10598309 & 0.10985423 & 0.11386954 & 0.11802337 & 0.12232122\end{array}$




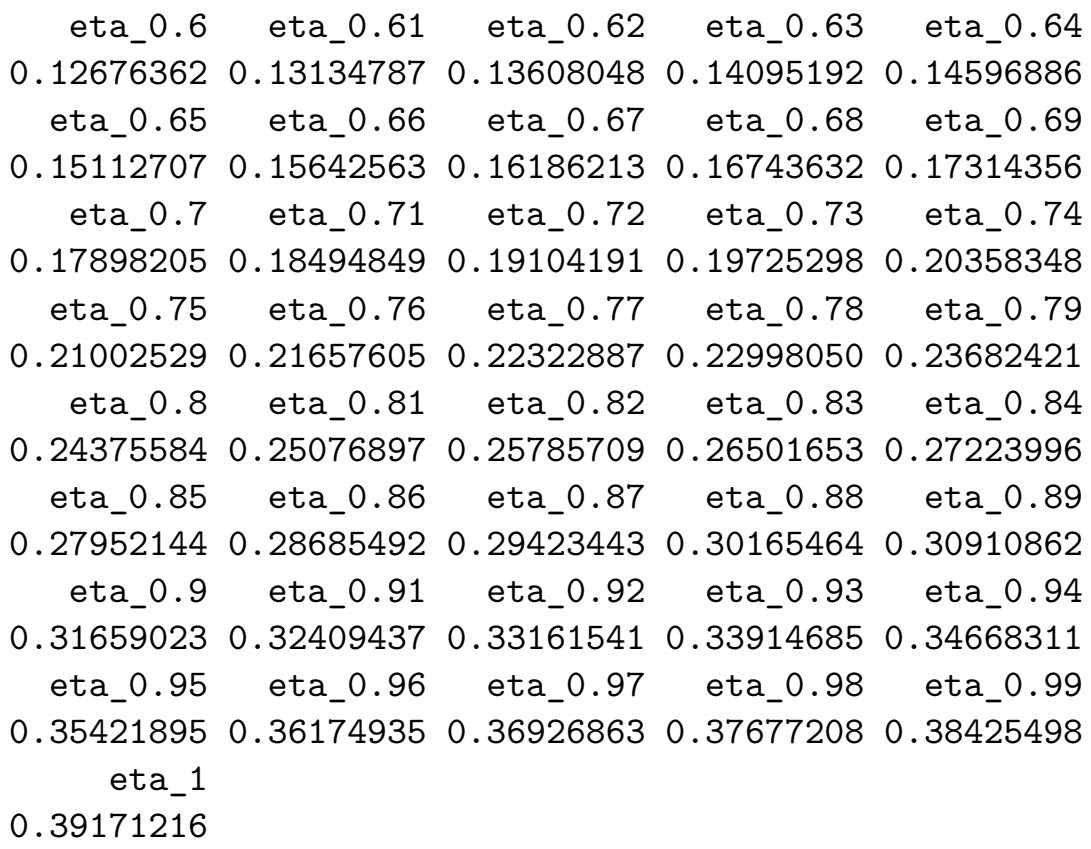

The test statistic takes its maximum for the score $(0,0,1)$ (as above), its corresponding adjusted $p$-value is only marginally larger compared to the MAX test based on three score vectors (0.03151 vs. 0.03042$)$.

\author{
Affiliation: \\ Ludwig A. Hothorn \\ Institut für Biostatistik \\ Leibniz Universität Hannover \\ Herrenhäuser Straße 2 \\ DE-30419 Hannover, Germany \\ Torsten Hothorn \\ Institut für Statistik \\ Ludwig-Maximilians-Universität München \\ Ludwigstraße 33 \\ DE-80539 München, Germany \\ E-mail: Torsten.Hothorn@R-project.org \\ URL: http://www.stat. uni-muenchen.de/ hothorn/
}

